\title{
A long overdue recognition
}

\author{
Arthur Ashkin has been awarded half of this year's Nobel Prize in Physics for his invention of optical tweezers.
}

O n 2 October it was announced that the 2018 Nobel Prize in Physics was being awarded "for groundbreaking inventions in the field of laser physics". One half of the award went to Arthur Ashkin "for the optical tweezers and their application to biological systems", and the other half jointly to Gérard Mourou and Donna Strickland "for their method of generating highintensity, ultra-short optical pulses" ${ }^{\text {. }}$

In 1970, while working at Bell Labs, Ashkin, the father of optical tweezers, reported in Physical Review Letters the acceleration and trapping of particles by radiation pressure ${ }^{2}$, and in 1987 he used the tweezers to capture living bacteria without harming them ${ }^{3}$. The rest is history. Optical tweezers are now routinely used in many fields of physical, chemical and biological sciences ${ }^{4}$.

The breaking news put optics and photonics, once again, in the Nobel limelight, following numerous other past recognitions ${ }^{5}$. The early morning call from Stockholm, Sweden, took Ashkin, 96, by surprise and made him the oldest Nobel Prize winner. In the interview with NobelPrize.org", he said "I didn't realize I am the oldest ever! So I just about made it. Because you can't be dead and win."

Indeed, Ashkin had been tipped to win the prize for many years. The response from the community is clear - it is a long awaited, well-merited recognition.

"I am very happy of the decision to award the Nobel Prize to Art Ashkin. I believe it is well deserved and long overdue. In my Nobel lecture, I described how Art played the pioneering role in particle trapping and one of the crucial roles in atom trapping," Steven Chu from Stanford University, USA told Nature Photonics. Chu worked with Ashkin at Bell Labs in the 1980s and is the co-recipient of the 1997 Nobel Prize in Physics "for development of methods to cool and trap atoms with laser light".

"The major impact of Art's work is that he introduced the physical basis to manipulate individual molecules and individual molecular assemblies. That allowed researchers to measure the forces and observe the dynamics between these molecules or individual molecules in a given environment. The method he introduced had especially important impacts in biophysics, biology and polymer physics," Chu further commented.

Kishan Dholakia from the University of St Andrews, UK, holds the same view as Chu: "I think it is richly deserved and long overdue.

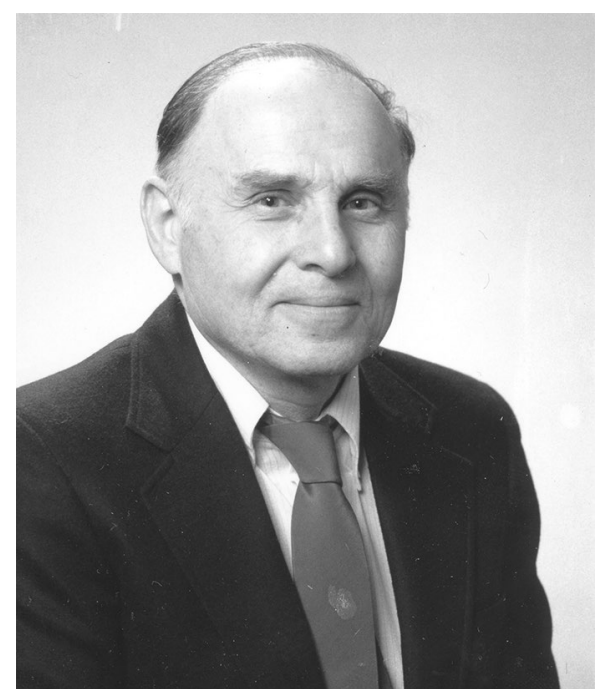

Credit: AIP Emilio Segrè Visual Archives, Physics Today Collection

I am absolutely thrilled for Arthur Ashkin as the impact this field has made since his first paper in 1970 is immense and shows no sign of abating." He continued, "this is clearly a topic that has caught the imagination of scientists across the spectrum and has been used for a myriad of studies. The breakthrough in physics this presents is reinforced by the fact that tweezers offer routes to exquisite control, motion and measurement that other approaches simply cannot match."

"Being able to control the position of small objects with light is fascinating. It impacts on numerous discoveries in physics, biology, material sciences and the list is only growing. And it all started with Arthur Ashkin's levitation trap," said Aristide Dogariu from the University of Central Florida, USA.

The idea of the mechanical action of light by pressure was first suggested in 1619 by Johannes Kepler, theoretically showed by James Clerk Maxwell in 1873, and experimentally proven by Pyotr Lebedev, Ernest Nichols and Gordon Hull in the early 1900s. It was not until the invention of the laser in 1960, which provided a more intense and directional light source, that the real utilization of optical forces could be realized.

"Starting from 1970, Arthur Ashkin has demonstrated in a series of papers that neutral particles, from atoms to micron-size, can be accelerated or in certain conditions even trapped by intense laser beams," said Dogariu. "These discoveries affected significantly our knowledge of the interplay between radiation and matter, and now, the truly revolutionary capability to manipulate the transfer of momentum from light to matter is recognized with a Nobel Prize."

Having worked in so many areas of photonics, Dholakia expressed that it's difficult for him to think of something comparable to optical trapping/tweezers that has had such a diverse and sustained range of world-class outputs across all of the sciences. "Quite simply it has clearly made an exceptional impact in so many fields for over forty years. We can use this beautiful noncontact method to hold single atoms, cells, colloids and viruses," said Dholakia.

Dholakia described that optical tweezers have become a powerful tool, for example, by using the angular momentum of light spin and orbital - and other more exotic light fields, such as propagation-invariant, Bessel and Airy beams ${ }^{7}$. Other key aspects include their combination with spectroscopic approaches, such as fluorescence and Raman spectroscopy, and extension to multiple traps for sorting and colloidal dynamics.

To Dogariu, tailoring the spatial and temporal characteristics of light is now opening unexplored and sometimes counterintuitive means for controlling nanoand microscopic objects. Indeed, new concepts have been demonstrated during the past few years for manipulating small objects using optical tractor beams - optically induced 'negative forces' that can exert a negative radiation pressure, or non-conservative force, on a body, thus pulling it towards the light source ${ }^{8}$. They can be achieved by tailoring the properties of the electromagnetic field, the environment or the particles themselves.

"Overall, I think the Nobel Prize will inspire a new generation of researchers in this constantly tantalizing, exciting field. I expect ever more surprising uses of this wonderful, elegant piece of physics. The best may be yet to come with optical tweezers!" said Dholakia.

Published online: 26 October 2018 https://doi.org/10.1038/s41566-018-0299-9

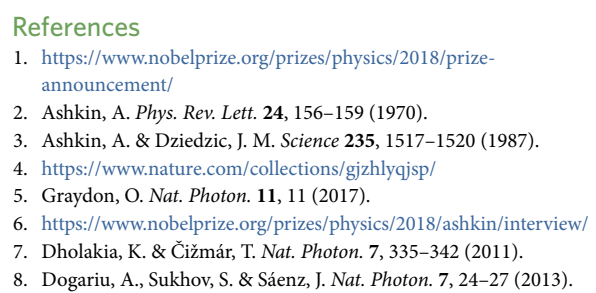

